\title{
ALLOCUTION DE M. FRANCKX AU NOM DU COMITE PROVISOIRE
}

Mr. Chairman, Mesdames et Messieurs,

C'est pour nous une très grande joie d'avoir, ici à New-York, à rendre compte aux membres d'Astis des résultats que nous avons obtenus depuis notre première réunion non officielle, lors du XIVe Congrès International à Madrid.

Nous vous rappellons que lors de cette séance privée, les membres présents ont exprimé le voeu que notre organisation puisse se constituer et s'intégrer comme partie véritable dans le sein du Comité Permanent des Congrès d'Actuaires.

A cette fin, un comité provisoire composé de Beard, Franckx, Johansen, Monic a été chargé de rechercher avec l'accord du Comité Permanent une solution qui permettrait, dans le cadre de ce Comité, de favoriser l'étude des problèmes de l'assurance des risques divers.

$\mathrm{Au}$ cours de ces trois années, de multiples contacts ont eu lieu à Bruxelles, à Londres et lors de réunions anniversaires à Zurich et à Edimbourg. Nous sommes vraiment heureux de pouvoir dire que de toute part on nous a encouragé dans nos efforts et que de tous les côtés nous avons été entendus avec intérêt et surtout avec le sincère désir d'arriver à une solution constructive.

Nous amenions une formule de travail et d'organisation nouvelle, mais il est bien vite apparu, que la création d'Astin comme section autonome devait entraîner une modification des statuts du Comité Permanent.

Des propositions en ce sens ont été introduites par Monsieur Marchand, Président de l'Association des Actuaires Suisses, et la section d'Astin ne pourrait exprimer assez sa gratitude à Monsieur Marchand, d'avoir catalysé par son intervention la formation d'Astin.

Ces propositions ont été admises par le Comité Directeur du Comité Permanent et seront soumises à l'Assemblée ici même à New York vendredi.

Simultanément le Comité d'Astin a fait un projet de règlement 
qui fut proposé au Président et au Secrétaire Général du Comité Permanent et soumis pour examen aux différentes organisations nationales.

Après rectifications ce règlement a été mis à l'ordre du jour de la séance du Comité Directeur qui a eu lieu ici même hier matin. Après approbation et conformément aux modifications des nouveaux statuts du Comité Permanent, nous avons eu la joie de voir Sir Georges Maddex accepter d'être auprès d'Astin le premier délégué du Comité. Sous son patronage et sa haute autorité, nous sommes convaincus que la nouvelle section d'AsTin établira dés ses débuts ses traditions dans une ligne qui sera un exemple pour d'autres sections à créer. Nous pensons que c'est ainsi que nous pourrons au mieux exprimer notre gratitude à Sir George.

Sous réserve de l'approbation du règlement du Comité Permanent, rien ne s'oppose à la création officielle d'Astin et c'est là l'objet de notre réunion d'aujourd'hui.

Mais avant de terminer permettez nous de remercier tous ceux qui ont effectivement contribué à la réalisation de ce grand jour.

Avant tous nos remerciements les plus chaleureux s'élèvent à MM. Théate, président du Comité Permanent et Boels, secrétaire général, qui tout au long de nos pourparlers ont oeuvrés et manoeuvrés pour éviter les embûches dressées devant nous, avec le sincère désir de nous aider dans l'accomplissement de notre but.

Nous nous devons de signaler le concours chaleureux qui nous a été accordé par les actuaires américains. Devons nous rappeler que Mr. Linton, président du comité organisateur de ce XVe congrès, nous a proposé de choisir le sujet IVA, qu'il nous a prêté tout le concours de son organisation et que tout fut mis en oeuvre pour qu'aujourd'hui dans les locaux de Commodore la séance d'Astın puisse avoir lieu. Nous avons aussi eu ce merveilleux appui de la Casualty Actuarial Society d'Amérique, qui sur le plan américain précède depuis longtemps l'action d'AstıN et nous accorde l'appui de toute son expérience. Nous soulignons ce geste de la Casualty Actuarial Society qui à l'occasion de notre formation a eu cette merveilleuse idée d'inviter à une très belle party tous ceux qui s'intéressent à nos efforts. Cela nous a permis de mieux nous connaître et de mieux nous comprendre et de sentir que la 
bonne volonté est partout, tant dans le vieux monde que de ce côté-ci de l'Atlantique.

Nous ne pourrions cacher que certaines compagnies ont voulu donner un appui financier dès la constitution d'AstiN et nous ont promis une contribution dont la valeur s'élève à $\$ 2.200$ et qu'un membre d'Astin a offert l'impression gratuite des travaux d'Astin pendant la Ie année de son existence.

Nous avons à excuser à cette réunion M. le Prof. Cantelli, président des actuaires Italiens, M. de Mori de Rome et M. Neuhaus de Zurich qui n'ont pu se joindre à nous.

Enfin au nom du Comité Provisoire nous remercions tous les membres de leurs encouragements. Aujourd'hui le comité peut considérer sa mission comme terminée; le but assigné est atteint.

Nous devons nous tourner vers l'avenir et cet avenir, chers amis, est entre vos mains. Astin sera ce que nous tous et surtout les jeunes vous en ferez. Nous avons créé un instrument d'action, pour servir, au sens le plus large du mot: servir, pour aider les hommes à surmonter les hasards du sort et du malheur, servir pour contribuer à l'avancement de la science pour promouvoir la recherche scientifique, pour unir nos efforts au service de tous.

C'est bien un idéal, mais les plus belles pensées qui ne se traduisent pas sur le plan des réalisations pratiques sont des fleurs sans fruit et Astin aura son importance dans la mesure où vous tous affirmerez par vos actions, par vos travaux que son existence est pleinement justifiée.

Il nous reste à souhaiter à tous bonne chance et bon travail. 